\title{
Errata
}

\section{Erratum: Radiative corrections to neutrino-induced neutral-current phenomena in the $\mathrm{SU}(2)_{L} \times \mathrm{U}(1)$ theory [Phys. Rev. D 22, 2695 (1980)]}

W. J. Marciano and A. Sirlin

We have found the following typographical errors:

(i) On the right-hand side of Eq. (B16) the first set of square brackets should be deleted and the symbol Re inserted before the second integral.

(ii) On the right-hand side of Eq. (A18b) a closing square bracket should be placed after $\ln \left(m_{Z} / \mu\right)$.

(iii) The subsection II F title should read " . . . $\bar{\nu}$-induced . . ",

Erratum: Some general properties of the $O(\alpha)$ corrections to parity violation in atoms [Phys. Rev. D 29, 75 (1984)]

W. J. Marciano and A. Sirlin

The muon decay constant below Eq. (17) should be $G_{\mu}=(1.16632 \pm 0.00002) \times 10^{-5} \mathrm{GeV}^{-2}$.

\section{Erratum: Testing the standard model by precise determinations of $W^{ \pm}$and $Z$ masses} [Phys. Rev. D 29, 945 (1984)]

William J. Marciano and A. Sirlin

Equation (14) should have properly read

$$
\delta \simeq \frac{11}{48 \pi} \frac{\alpha}{\sin ^{2} \theta_{W}} \ln \left(\frac{m_{\phi}^{2}}{m_{Z}^{2}}\right)+\cdots, m_{\phi}^{2} / m_{Z}^{2}>>1 .
$$

